\title{
Contributors to the volume and their entries
}

Aldo Ahlers Jr, Rollins College, Winter Park, FL, USA

Sun Guangxin

Matthew Amick, Walt Disney, Orlando, FL, USA

Chang Chung-Mou (Zhang Zhongmou), Jiang Nanchun (Jason Jiang), Barry Lam

(Lin Baili), Wen Sayling (Wen Shiren), Wu Yijian

Dongmei Cao, College of Charleston, Charleston, SC, USA

Chen Jinyi, Liang Jianzhang, Wu Ying, Zhao Xinxian, Zhu Mengyi

Julian Chambliss, Rollins College, Winter Park, FL, USA

Bao Yugang, Chai Songyue, Feng Lun, Henry Ying Tung Fok (Huo Yingdong), Shen Taifu

Howard Chiang, Princeton University, Princeton, NJ, USA

Zhang Weiying

Victoria Chu, University of California, San Diego, CA, USA

Ma Hong, Ning Xiangdong, Wu Dingfu, Xie Fuzhan, Xie Xuren

Mary Conway Dato-on, Rollins College, Winter Park, FL, USA

Li Rucheng, Henry Sy Sr (Shi Zhicheng), Yang Rong, Zheng Yonggang

Huijuan Cui, Renmin University of China, Beijing, China

Ma Weihua

Xiaodong Du, Rollins College, Winter Park, FL, USA

Guo Zeli, Ni Runfeng

Quek Kia Fatt, Monash University, Sunway, Malaysia

William Hengjem Cheng (Zhong Tingshen), Hu Maoyuan, Robert Hock Nien Kuok (Guo Henian), Yang Guoping, Yin Tongyao

Marc Fetscherin, Rollins College, Winter Park, FL, USA

Chen Lihua, Gu Chujun, Guo Hao, Li Xiaohua, Liu Hanyuan, Sun Guangxin, Xu Ming, Xu Rongmao, Zhang Simin

Mareike Fetscherin, Rollins College, Winter Park, FL, USA

Henry Ying Tung Fok (Huo Yingdong), Shen Taifu

Charlotte Froehlich, Rollins College, Winter Park, FL, USA

Chen Lihua

Daniel Galvez, Rollins College, Winter Park, FL, USA

Xu Ming

Adeta Gayah, Rollins College, Winter Park, FL, USA

Li Xiaohua 
James P. Gilbert, Rollins College, Winter Park, FL, USA

Li Lihui, Wang Xuebing, Yi Gang, Zhang Enzhao, Zhang Jianguo, Zhou Xiaochuan

Hui He, George Washington University, Washington, DC, USA

Li Jun, Li Yanhong, Rong Yiren, Wu Yi, Xiang Huaicheng

Alex Hellberg, Rollins College, Winter Park, FL, USA

Liu Hanyuan

Arthur Holst, City of Philadelphia, PA, USA

Chen Feng, Duan Qiang, Ma Wanqi (Ma Man-Kei), Mu Xinsheng, Wei Jiafu

Lujin Huang, East China University of Science and Technology, Shanghai, China

Cao Guowei, Chang Xing, Chen Xiao, Chen Xiaoxian, Ding Jian, Fan Gang, Gao Dekang,

Jing Shuping, Pan Shiyi, Song Weiping, Wen Tiejun, Xie Tielan, Xu Xiaoping, Yang

Kaisheng, Yang Mingsheng, Yu Shumin, Yu Yu, Zhang Chaoyang (Charles Zhang), Zheng Shengtao, Zhou Bohua

Loi Teck Hui, University of Malaya, Kuala Lumpur, Malaysia

William Hengjem Cheng (Zhong Tingshen), Hu Maoyuan, Robert Hock Nien Kuok (Guo Henian), Yang Guoping, Yin Tongyao

David Irving, Rollins College, Winter Park, FL, USA

Chai Songyue, Feng Lun

Jonatan Jelen, New School University, New York, NY, USA

Miao Shouliang, Peng Xiaofeng, Sun Hongbin, Yang Guoqiang, Ye Lipei (Eddie Ye), Zhang Jindong

Hong Jiang, Renmin University of China, Beijing, China

Yang Yuanqing

Qian Hu, Renmin University of China, Beijing, China

Li Dongsheng

Thomas D. Lairson, Rollins College, Winter Park, FL, USA

Chen Yun, Deng Xiaoping, Wen Jiabao, Zhao Ziyang, Zhu Rongji

Christoph Lattemann, Potsdam University, Potsdam, Germany

Huang Guangyu, Liu Yonghao, Lu Guanqiu, Nan Cunhui

Jen-Kai Liu, German Institute of Global and Area Studies, Hamburg, Germany

Bo Xilai, Xu Kuangdi, Yang Yuanyuan, Zhang Qingwei, Zhu Yanfeng

Francisco Lutz, Rollins College, Winter Park, FL, USA

Gu Chujun

Michael J. Miske, Dawson \& Clark, Detroit, MI, USA

Fu Chengyu, Jin Zhiguo, Li Shufu, Shang Fulin, Tian Deju

Xiafang Mo, Freelance writer, Beijing, China

Li Yining, Ma Yun, Wang Shi, Wu Jinglian, Yang Lan 
Michael A. Moodian, Chapman University, Orange, CA, USA

Li Zekai (Richard Li, Tzar Kai Li), Yang Bin, Yuan Longping, Zhang Yin, Zhou Zhengyi

Rebecca Montaner, Rollins College, Winter Park, FL, USA

Geng Xiaoping

Guibin Mu, Renmin University of China, Beijing, China

Zhou Houjian

Tim Poplin, Rollins College, Winter Park, FL, USA

Zhang Simin

Natalie Powers, Rollins College, Winter Park, FL, USA

Xu Rongmao

Martina Jing Quan, University of Texas, Dallas, TX, USA

Li Ning, Ma Huateng, Mai Boliang, Ren Zhengfei, Wang Wenjing

Hui Shi, Renmin University of China, Beijing, China

Dong Mingzhu, Ning Gaoning

Amir Shoham, College of Management, Tel Aviv, Israel

Li Jun, Li Yanhong, Rong Yiren, Wu Yi, Xiang Huaicheng

Sangeeta Singh, BI Norwegian School of Management, Oslo, Norway

Ding Lei, Guo Guangchang, Li Ka Shing (Li Jiacheng), Liu Chuanzhi, Zong Qinghou

Linda G. Sprague, Rollins College, Winter Park, FL, USA

Liu $\mathrm{Ji}$

Clay Stanfield, Rollins College, Winter Park, FL, USA

Dai Zhikang, Liu Bo, Liu Xiaoguang

David Straub, Indiana University, Bloomington, IN, USA

Eysayup Ekrem (Aikelamu Aishayoufu), Rebiya Kadeer, Mayirjon (Mayierjiang), Mi Enhua, Tang Wanxin

Jianmin Sun, Renmin University of China, Beijing, China

Dong Mingzhu, He Boquan, Hou Weigui, Li Dongsheng, Ma Weihua, Ning Gaoning, Xie

Qihua, Yang Yuanqing, Ye Long, Zhang Yue, Zhou Houjian

Sunny Li Sun, University of Texas, Dallas, TX, USA

Li Ning, Ma Huateng, Mai Boliang, Ren Zhengfei, Wang Wenjing

Shawn Tavares, Rollins College, Winter Park, FL, USA

Guo Hao

Siri Terjesen, Indiana University, Bloomington, IN, USA

Zhang Gang

Rachel Todd, Rollins College, Winter Park, FL, USA

Donald Tsang (Zeng Yinquan)

Min Tong, University of Central Florida, Orlando, FL, USA

Chi Yufeng, Guo Xianchen, Mu Zhanying, Pan Ning, Wang Weibin 
Fu-Sheng Tsai, Cheng Shiu University, Kaohsiung, Taiwan

Steve Chang (Zhang Mingzheng), Terry Kuo (Guo Taiming), Lee Rie-Ho (Li Ruihe),

Stan Shi (Shi Zhenrong), Jerry Chih-Yuan Yang (Yang Zhiyuan)

Denise Tsang, University of Reading, Reading, UK

Chen Tianqiao, Stanley Ho (He Hongshen), Liu Jiren, Qiu Bojun, Xu Shaochun

Hua Wang, Euromed Marseille Ecole de Management, Marseille, France

Liu Changping, Miao Wei, Tan Xuguang, Wang Linxiang, Wang Yung-ching (Wang Yongqing)

Zhen Wang, Renmin University of China, Beijing, China

Ye Long

Joshua Wickerham, University of California, San Diego, CA, USA

Hu Shuli, Li Jinhua, Li Xiaopeng, Wang Mengkui, Wang Zhidong

Audrey Wu, China Europe International Business School, Shanghai, China

Liu Ji

Depin Yang, Renmin University of China, Beijing, China

Zhang Yue

Tao Yang, Renmin University of China, Beijing, China

He Boquan, Hou Weigui

Shirley Ze Yu, Yu Financial, Altamonte Springs, FL, USA

Li Jingwei, Long Yongtu, Shi Xiaoyan, Tsang Hin Chi (Zeng Xianzi), Wang Yan

Xiaoqi Yu, Kent State University, Kent, OH, USA

Duan Yongping, Ge Wenyao, Kong Dan, Shaw Runrun (Shao Yifu), Wang Liheng

Hong Zhang, University of Central Florida, Orlando, FL, USA

Lang Xianping, Shi Yuzhu, Wang Guangying, Wang Jiafen, Yu Zuomin

Jian Zhang, University of Hawaii at Manoa, Honolulu, HI, USA

Hu Angang, Rong Zhijian

Weidong Zhang, Winona State University, Winona, MN, USA

Lai Changxing, Mou Qizhong, Nian Guangjiu, Shi Zhengrong, Wang Xuan

Wenxian Zhang, Rollins College, Winter Park, FL, USA

Chang Xiaobing, Li Xiaopeng, Donald Tsang (Zeng Yinquan), Wang Mengkui, Wang Zhidong, Wang Zhongjun, Wu Renbao, Zhang Ruimin

Ying Zhang, University of Central Florida, Orlando, FL, USA

Bi Dongjiang, Deng Zhonghan, Pan Gang, Tian Suning, Zhou Dahu

Yi Zhao, Renmin University of China, Beijing, China

Xie Qihua 